\title{
A NEW APPROACH IN CRASHING AND INTRODUCTION OF $P$ FACTOR
}

\author{
Kanika Mandal ${ }^{1}$, Kajla Basu ${ }^{2}$ \\ ${ }^{1,2}$ Department of Mathematics \\ NIT Durgapur \\ West Bengal, 713209, INDIA
}

\begin{abstract}
Crashing a project means crashing a number of activities to reduce the duration of the project below its normal time. To avoid unfavorable circumstances or to be in competition with other competitors who are to launch same type of products, Crashing is helpful. Because of the uncertainties in daily life, the time duration or the cost involved for the activities in a network can not be known in a precise manner. To deal with the vagueness or imprecise data, it is required to use fuzzy time and cost for activities in a network. A new approach for crashing the project with fuzzy activity times and cost is introduced in this paper. A study has been carried out to improve the probability of achieving the target time. In doing so a new probability factor called $\mathrm{p}$ factor has been introduced in the paper for the first time. This $\mathrm{p}$ factor will also help the decision maker to choose his own target time. The new approach is efficient and easy to implement for a real life situation. The approach has been discussed step by step theoretically and finally presented with a numerical example.
\end{abstract}

AMS Subject Classification: 90B99, 90B50

Key Words: fuzzy critical pathm crashing, probability factor

\section{Introduction}

Crashing in project scheduling is important to avoid unfavorable situations like bad weather, to launch products first in the market amongst competitors.

Received: $\quad$ May 12, 2016

Revised: July 25, 2016

Published: November 5, 2016

$\S_{\text {Correspondence author }}$ (c) 2016 Academic Publications, Ltd. url: www.acadpubl.eu 
Crashing requires extra budget (for extra labor cost, quick procurement of materials even in high price and other related extra cost to quicken the process and activities).

In [2], [3], [4] fuzzy numbers are used as activity time to determine critical path. Some researchers worked on crashing and project completion probability. In [13] Priti Singh, Florentin Smarandache et al. proposed an algorithm for unit crashing that reduces the cost of project. Also $C^{++}$program was developed to achieve their results. Ajiboye Sule Adegoke [14] showed how CPM/PERT can be applied to solve quality problems in an organization. They also used CPM/PERT to measure process effectiveness. Komesh Sahu and Meenu Sahu [15] gave an alternative method of approach to obtain optimum cost and time and also the minimum duration of project with fully crashing in critical path. P.K. Suri and Bharat Bhushan solved the cost and scheduling problem of software project using PERT network in [16]. With the help of crashing they reduced software project duration at a minimum cost by locating a minimal cut in the duration of an activity of the original project design network.

But no work has been done till date on crashing in fuzzy network. In this paper we have first shown how a project can be crashed in a realistic manner using fuzzy activity time and fuzzy cost to express uncertainty and vagueness about the time duration and cost for activities. Moreover, it is obvious that the variation of probability occurs to meet the reduced time before and after the crashing (with the new approach using fuzzy) due to variation in the activity completion time. If the probability to meet the reduced target time after crashing is not acceptable to project management or they want the probability to be in higher side, we introduce a new factor called the probability factor or p-factor, to increase it. Every project management normally sets the target time depending on many decision factors and does not want to miss the target time. So they want probability to meet the target time at higher side. The p-factor newly introduced in this paper can bring the probability to expected range.

The p-factor is designed in such a way that its range can be calculated for a project as well as probability and cost variation can be derived using it. So project management can choose the probability value as per their extra budget and the corresponding p-factor. To be precise, we have introduced a new realistic approach of crashing using fuzzy time and cost as well as a new probability factor ( $\mathrm{p}$-factor) to increase the probability of meeting the target time to a desired value within extra budget of a project.

This paper is organized as follows: section 2 gives an introduction of Fuzzy sets, operations on them and their ranking, Section 3 briefly describes project 
completion probability, section 4 introduces a practical approach of crashing step by step using fuzzy time and cost for activities as well as a new factor called the p-factor to increase the project completion probability to a higher acceptable value and in section 5 a numerical example is presented to show how the activities of a project network can be crashed realistically and how the probability to finish the project earlier can be improved. In Section 6 conclusion of the paper has been presented.

\section{Fuzzy Sets}

A fuzzy set can be mathematically constructed by assigning to each possible individual in the universe of discourse a value representing its grade of membership in the fuzzy set . This grade corresponds to the individuals similarity to the concept represented by the fuzzy set. The fuzzy number $\tilde{A}$ is a fuzzy set whose membership function $\mu_{\tilde{A}}(x), \mu$ satisfies the following conditions:

(i) $\mu_{\tilde{A}}(x)$ is piecewise continuous;

(ii) $\mu_{\tilde{A}}(x)$ is a convex fuzzy subset;

(iii) $\mu_{\tilde{A}}(x)$ is the normality of a fuzzy subset, implying that for at least one element $x_{0}$ the membership grade must be 1 , i.e. $\mu_{\tilde{A}}\left(x_{0}\right)=1$.

\subsection{Definition}

A fuzzy number with membership function in the form

$$
\mu_{\tilde{A}}(x)= \begin{cases}\frac{x-a}{b-a}, & \text { if } a \leq x \leq b \\ \frac{c-x}{c-b}, & \text { if } b \leq x \leq c \\ 0, & \text { otherwise }\end{cases}
$$

is called a triangular fuzzy number $\tilde{A}=(a, b, c)$.

\subsection{Operation on Triangular Fuzzy Number}

The following are the four operations that can be performed on triangular fuzzy numbers: Let $\tilde{A}=\left(a_{1}, a_{2}, a_{3}\right)$ and $\tilde{B}=\left(b_{1}, b_{2}, b_{3}\right)$ then:

(i) Addition: $\tilde{A}+\tilde{B}=\left(a_{1}+b_{1}, a_{2}+b_{2}, a_{3}+b_{3}\right)$. 
(ii) Subtraction: $\tilde{A}-\tilde{B}=\left(a_{1}-b_{3}, a_{2}-b_{2}, a_{3}-b_{1}\right)$.

(iii) Multiplication: $\tilde{A} \times \tilde{B}=\left(\min \left(a_{1} b_{1}, a_{1} b_{3}, a_{3} b_{1}, a_{3} b_{3}\right), a_{2} b_{2}, \max \left(a_{1} b_{1}, a_{1} b_{3}\right.\right.$, $\left.\left.a_{3} b_{1}, a_{3} b_{3}\right)\right)$.

(iv) Division: $\frac{\tilde{A}}{\tilde{B}}=\left(\min \left(\frac{a_{1}}{b_{1}}, \frac{a_{1}}{b_{3}}, \frac{a_{3}}{b_{1}}, \frac{a_{3}}{b_{3}}\right), \frac{a_{2}}{b_{2}}, \max \left(\frac{a_{1}}{b_{1}}, \frac{a_{1}}{b_{3}}, \frac{a_{3}}{b_{1}}, \frac{a_{3}}{b_{3}}\right)\right)$.

\subsubsection{Inversion Operation on Triangular Fuzzy Number}

The addition and subtraction of fuzzy numbers are not reciprocal operations, i.e., it is not possible to solve inverse problems exactly using the standard fuzzy arithmetic operators. To overcome this in function principle operation of triangular fuzzy number a new operation was proposed by A. Nagoor Gani in [12] that allows the exact inversion. That new operation is stated below:

Let $\tilde{A}=\left(a_{1}, a_{2}, a_{3}\right)$ and $\tilde{B}=\left(b_{1}, b_{2}, b_{3}\right)$, then $\tilde{A}-\tilde{B}=\left(a_{1}-b_{1}, a_{2}-\right.$ $\left.b_{2}, a_{3}-b_{3}\right)$. The new subtraction operation exist only if the following condition is satisfied: $D P(\tilde{A}) \geq D P(\tilde{B})$, where $D P(\tilde{A})=\frac{a_{3}-a_{1}}{2}$ and $D P(\tilde{B})=\frac{b_{3}-b_{1}}{2}$; DP denotes Difference point of a Triangular fuzzy number.

\subsection{Ranking of Triangular Fuzzy Numbers}

Let $\tilde{A}=\left(a_{1}, b_{1}, c_{1} ; w\right)$ and $\tilde{B}=\left(a_{2}, b_{2}, c_{2} ; w\right)$ are two triangular fuzzy numbers. Then rank (A) is defined as $\frac{a_{1}+7 b_{1}+c_{1}}{9} \times \frac{4 w}{9}$ as discussed in [1]. Now to compare $\tilde{A}$ and $\tilde{B}$ :

(i) If $\operatorname{rank}(\tilde{A})>\operatorname{rank}(\tilde{B}), \tilde{A} \succ \tilde{B}$;

(ii) If $\operatorname{rank}(\tilde{A})<\operatorname{rank}(\tilde{B}), \tilde{A} \prec \tilde{B}$.

\subsubsection{Example}

Let $\tilde{A}=(3,5,7 ; 1)$ and $\tilde{B}=(4,5,7 ; 1)$. Then $\operatorname{rank}(\mathrm{A})=2.222$ and $\operatorname{rank}(\mathrm{B})=$ 2.2716. Since $\operatorname{rank}(\tilde{A})<\operatorname{rank}(\tilde{B}) \Rightarrow \tilde{A} \prec \tilde{B}$

\section{Project Completion Probability}

PERT is a management technique to estimate the probability that a project will be finished on normal time. According to the traditional PERT technique the probability of a certain project meeting a specific schedule time can be described as follows:

$$
Z=\frac{T_{d}-T_{e}}{\sigma_{r}}
$$


Here:

i) $\mathrm{Z}$ is the number of standard deviations of the due date or target date lies away from the mean or expected date;

ii) $T_{e}$ is the normal expected time which is equal to the sum of normal expected times of activities on critical path. That means if $t_{1}, t_{2}, \ldots, t_{k}$ are the expected times of critical path activities, then $T_{e}=\sum_{i=1}^{k} t_{i}, i=1,2, \ldots, k$;

iii) $T_{d}$ is the due date or targeted date of completion; and

iv) $\sigma_{r}$ is the project standard deviation which is written as

$$
\begin{aligned}
\sigma_{r} & =\sqrt{\text { project variance }} \\
& =\sqrt{\sum \text { variance of activities on critical path }} \\
& =\sqrt{\sum_{i=1}^{k} v_{i}, \quad i=1,2, \ldots k .}
\end{aligned}
$$

where $t_{i}=\frac{a+4 m+b}{6}$ and $v_{i}=\left(\frac{b-a}{6}\right)^{2}$.

The time estimates $\mathrm{a}, \mathrm{m}, \mathrm{b}$ are defined as follows:

Optimistic time (a): the minimum possible time required to accomplish a task, assuming everything proceeds better than is normally expected.

Most likely time $(\mathrm{m})$ : the best estimate of the time required to accomplish a task, assuming everything proceeds as normal.

Pessimistic time (b): the maximum possible time required to accomplish a task, assuming everything goes wrong.

\section{Algorithm of New Approach in Crashing and Introduction of a New Factor to Increase the Probability of the Project Completion within Target Time}

\subsection{Introduction of p-Factor}

Probability factor or p-factor can be defined as a singleton whose triangular fuzzy representation is ( $p, p, p)$. When $\mathrm{p}$ factor is multiplied with a fuzzy activity time in a network, expected project completion time will be reduced resulting in increased probability (to meet the target time). 


\subsubsection{Range of p-Factor}

Case I: When after crashing all paths of a network are having same total activity time:

If p-factor is multiplied with every activity of critical path, then from equation (1) $Z_{n e w}=\frac{T_{d}-p T_{e}}{p \sigma_{r}}$, where $T_{e}$ and $\sigma_{r}$ are the normal expected time and project standard deviation after crashing respectively. From normal distribution table we see that probability increases whenever values of $z$ increases and at about $z=5$ probability reaches 0.999997 (nearly 1 ). So to increase the probability to meet the target time after crashing,

$Z_{\text {new }}>Z_{\text {old }}$, i.e. $\frac{T_{d}}{p \sigma_{r}}-\frac{T_{e}}{\sigma_{r}}>\frac{T_{d}}{\sigma_{r}}-\frac{T_{e}}{\sigma_{r}}$, i.e. The value of $\mathrm{p}$-factor should be less than 1.If $p=1$, equation becomes same as equation (1). so maximum value of p-factor is 1 . Considering $z=5$ (when the probability reaches 0.999997) from equation (1), we get the minimum value of $p=\frac{T_{d}}{5 \sigma_{r}+T_{e}}$. So the range of $\mathrm{p}$ will be $\frac{T_{d}}{5 \sigma_{r}+T_{e}} \leq$ pfactor $\leq 1$.

Case II: When both critical and non critical paths exists after crashing, determine the second largest path or the path where time consumption is second largest. Say the defuzzified time for this non-critical path is $T_{n}$ and critical path is $T_{m}$. It is obvious that the value of p-factor is less than 1 . Now we can choose $\mathrm{p}$ factor in such a way that by multiplication of $\mathrm{p}$ factor with every activity of critical path after crashing the defuzzified value of new expected time should not less than $T_{n}$, i.e. we can not choose $\mathrm{p}$ factor which is less than $\frac{T_{n}}{T_{m}}$, i.e. if every activity of critical path is multiplied by $p=\frac{T_{d}}{5 \sigma_{r}+T_{e}}$ and the resulting new expected time is greater than $T_{n}$, then minimum value of $\mathrm{p}$ factor is $\frac{T_{d}}{5 \sigma_{r}+T_{e}}$, otherwise $\frac{T_{n}}{T_{m}}$, i.e. range will be maximum of $\left(\frac{T_{d}}{5 \sigma+T_{e}}\right.$ and $\left.\frac{T_{n}}{T_{m}}\right) \leq \mathrm{p}$ factor $\leq 1$.

\subsection{Algorithm}

Step 1: Determine the normal project completion time and associated critical path. To compare fuzzy numbers, meaning, to determine which one is greater, follow the rule defined in subsection 2.3.

Step 2: Calculate the cost slope for each activity which is relevant for crashing, i.e. cost slope $=\frac{C_{c}-C_{n}}{T_{n}-T_{c}}$. where $C_{c}=$ Fuzzy crash cost , $C_{n}=$ Fuzzy normal cost, $T_{n}=$ Fuzzy normal time, $T_{c}=$ Fuzzy crash time.

Step 3: For reducing the total project completion time, identify and crash an activity time on the critical path with lowest cost-slope value to the point where: 
(i) another path in the network becomes critical or

(ii) the activity has been crashed to lowest possible time.

Step 4: If the critical path under crashing is still critical, return to step 3. However, if due to crashing of an activity time in step 3, other paths in the network also become critical, then identify and crash the activity(s) on the critical path(s) with minimum cost slope.

Step 5: Terminate the procedure when each critical activity has been crashed to its lowest possible time. Determine the crashed time of the project.

Step 6: Determine the probability (before and after crashing) to complete the project considering the crashed time as the target time. Determine the probability as stated in section 3 .

Step 7: It can be observed that probability (to meet the target time) before crashing $<$ probability after crashing. The reason is that extra cost spent due to crashing modifies the project critical activity time variation. To increase the probability after crashing to a higher value, use p-factor which is discussed in subsection 4.1 .

Step 8: Prepare a table with the probability for different values of p-factor and the corresponding extra cost. Probability $\left(Z=\frac{T_{d}-p T_{e}}{p \sigma_{r}}\right)$ and Extra cost per critical path $=\left(\right.$ Critical path duration $\left(T_{c p}\right)-(\mathrm{p}, \mathrm{p}, \mathrm{p}) \times$ Critical path duration $\left.\left(T_{c p}\right)\right) \times$ cost per day of the critical path decided by the organization to increase probability $\left(c_{c p}\right)$.

\section{Numerical Example}

An organization $O_{1}$ wants to launch a certain product in a specific country matching product capabilities to market needs. They have to perform some activities before launch it to the market like gathering the requirements specific to the location (the size/strength/price etc. which the people of the area can afford), establishment of a plant, Designing the product, procurement of raw materials and preparation of the decided number of products. The normal cost and time for the different activities is given in table 1. The organization, after doing some market study, found that if the product could be launched sometime earlier, it would be better to maximize sales and profit. The related crash cost and time to launch it earlier is also given in table 1. And moreover, 
the organization wants the probability value to meet the target time at higher side so that the chance to miss the earlier date is less. The organization decides total (in USD) $(1000,2000,4000)$ and $\operatorname{cost}(\mathrm{USD})$ per day $(150,250,400)$ for this purpose (to increase probability to finish the project earlier). Project network with its activities is shown in Fig 1.

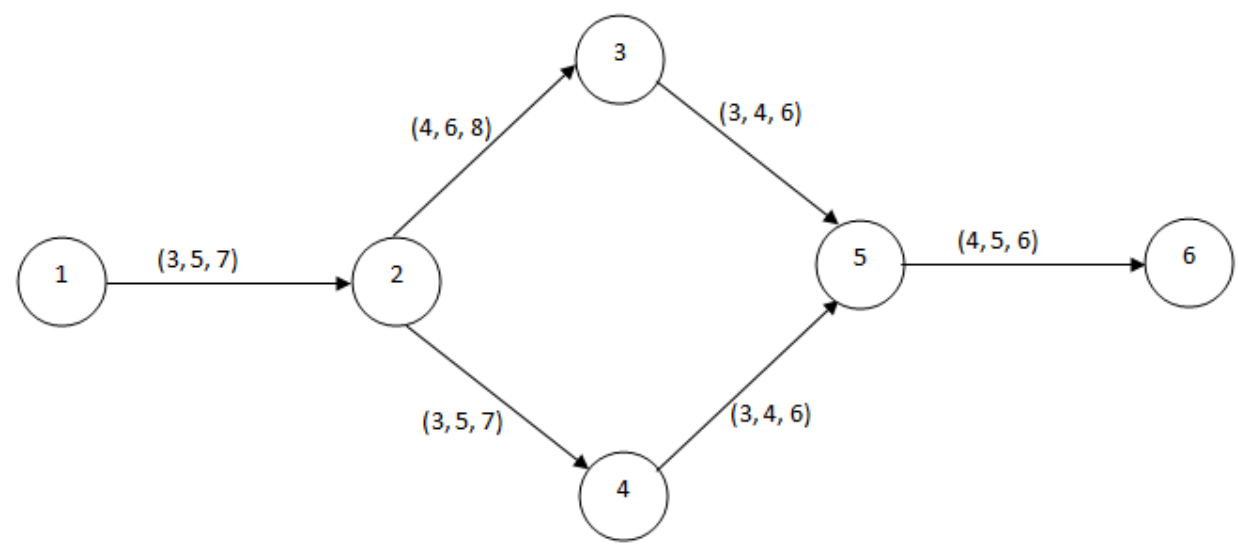

Figure 1: A Fuzzy Project Network before crashing.

\begin{tabular}{|l|l|l|l|l|}
\hline Activity & $\begin{array}{l}\text { Normal time } \\
\text { (days) }\end{array}$ & $\begin{array}{l}\text { Normal cost } \\
\text { (USD) }\end{array}$ & $\begin{array}{l}\text { Crash time } \\
\text { (days) }\end{array}$ & $\begin{array}{l}\text { Crash cost } \\
\text { (USD) }\end{array}$ \\
\hline $1-2$ & $(3,5,7)$ & $(2000,3000,3500)$ & - & - \\
\hline $2-3$ & $(4,6,8)$ & $(1000,1200,1300)$ & $(2,2.5,3)$ & $(1400,1500,1600)$ \\
\hline $3-5$ & $(3,4,6)$ & $(900,1000,1050)$ & $(1,1.5,2)$ & $(1100,1200,1300)$ \\
\hline $2-4$ & $(3,5,7)$ & $(1100,1200,1250)$ & $(1,1.5,2)$ & $(1300,1400,1500)$ \\
\hline $4-5$ & $(3,4,6)$ & $(1000,2000,3000)$ & - & - \\
\hline $5-6$ & $(4,5,6)$ & $(1500,1600,1650)$ & - & - \\
\hline
\end{tabular}

Table 1: Normal as well as crash cost and time.

\subsection{Method of Solution}

Step 1: We can find the critical path from the defuzzified values of slack times calculated in Table 2. Critical path is 1-2-3-5-6 and normal duration $=(14,20,27)$. Also the total duration along the path 1-2-4-5-6 is $(13,19,26)$.

Step 2: Compute the cost slope using Fuzzy division operator (table 3).

Step 3: Since critical activity 3-5 has the minimum cost slope $(10,80,400)$ (as its rank is the least),it is first selected for crashing. We can crash activity 


\begin{tabular}{|l|l|l|l|}
\hline Activity & Fuzzy activity time & Slack time $(\mathrm{a}, \mathrm{b}, \mathrm{c})$ & Defuzzified value $\frac{a+b+c}{3}$ \\
\hline $1-2$ & $(3,5,7)$ & $(-13,0,13)$ & 0 \\
\hline $2-3$ & $(4,6,8)$ & $(-13,0,13)$ & 0 \\
\hline $3-5$ & $(3,4,6)$ & $(-13,0,13)$ & 0 \\
\hline $2-4$ & $(3,5,7)$ & $(-12,1,14)$ & 1 \\
\hline $4-5$ & $(3,4,6)$ & $(-12,1,14)$ & 1 \\
\hline $5-6$ & $(4,5,6)$ & $(-13,0,13)$ & 0 \\
\hline
\end{tabular}

Table 2: Defuzzified values of slack time.

\begin{tabular}{|l|l|l|l|l|l|l|}
\hline Activity & $1-2$ & $2-3$ & $3-5$ & $2-4$ & $4-5$ & $5-6$ \\
\hline $\begin{array}{l}\text { Cost Slope } \\
\text { (USD/day) }\end{array}$ & - & $(16.67,85.714,600)$ & $(10,80,400)$ & $(8.33,57.14,400)$ & - & - \\
\hline
\end{tabular}

Table 3: Cost slope of each activity.

3-5 maximum by $[(3,4,6)-(1,1.5,2)]=(2,2.5,4)$ (using subtraction rule defined in 2.2.1). But if we crash activity $3-5$ by $(1,1,1)$, then $1-2-3-5-6$ and $1-2-4-5-6$ both will be critical path, so crash activity $3-5$ by $(1,1,1)$; [since $\operatorname{rank}(1,1,1)$ $\leq \operatorname{rank}(2,2.5,4)]$. crash cost is $(1,1,1) \times(10,80,400)=(10,80,400)$ and project duration $=(13,19,26)$.

Step 4: In similar way next we crash activity $3-5$ and $2-4$ by $(1,1.5,3)$. So, crash cost $=(10,80,400)+(1,1.5,3) \times(10,80,400)+(1,1.5,3) \times(8.33,57.14,400)$ $=(28.33,285.71,2800)$ and project duration $=(12,17.5,23)$.

Activity 2-4 among the rest has minimum cost slope and can still be crashed by $(1,2,2)$. Here we transformed triangular fuzzy number $(1,2,2)$ into $(1-\epsilon, 2,2+\epsilon)$ to make it meaningful with both having same defuzzyfied value; where $\epsilon$ tends to zero. Crash activity $2-4$ and $2-3$ by $(1-\epsilon, 2,2+\epsilon)$.

Step 5: The project can not be crashed further (fig 2). crash cost = $(28.33,285.71,2800)+(1-\epsilon, 2,2+\epsilon) \times(8.33,57.14,400)+(1-\epsilon, 2,2+\epsilon) \times$ $(16.67,85.71,600)=(53.33,571.41,4800)$ and minimum total time $=(11,15.5,21)$.

Step 6: Now we will calculate the probability to meet the target time. We consider three target times for probability calculation purpose:

(a) 15 days, less than 15.67 , which is the expected value of project completion time after crashing,

(b) 13 days, more less than that expected value, and

(c) 16 days, a little bit greater than that expected value.

Table 4 and 5 shows the probability values before and after crashing respectively to meet the above mention target times. 


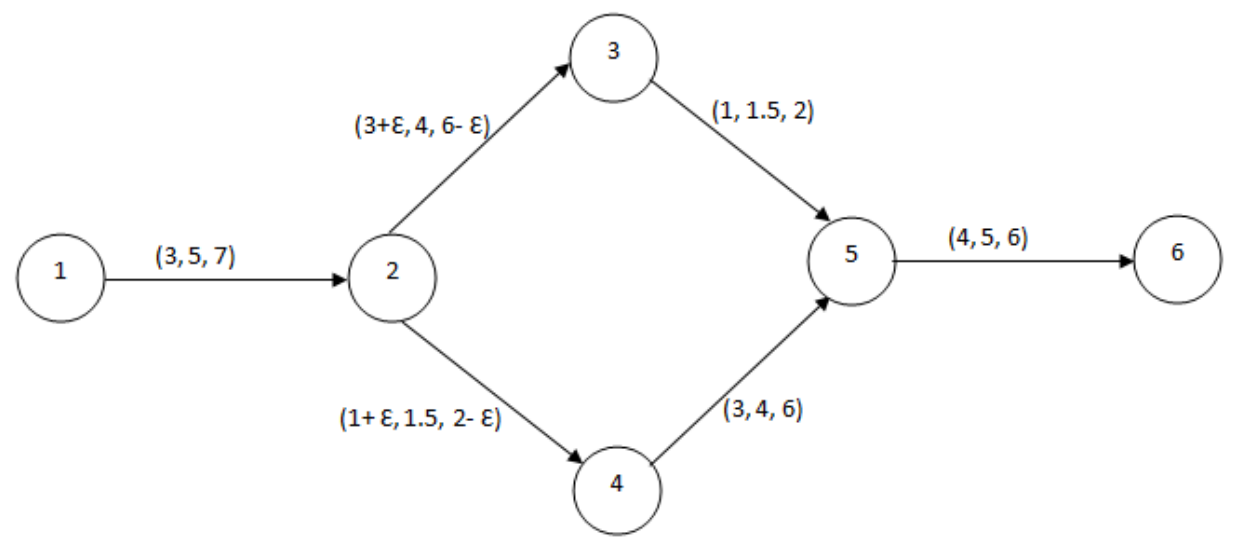

Figure 2: A Fuzzy Project Network after crashing.

\begin{tabular}{|l|l|l|l|l|}
\hline$T_{e}$ & $\sigma$ & $T_{d}$ & $Z$ & probability \\
\hline 20.17 & 1.118 & 15 & -4.62 & 0.000003 \\
\hline 20.17 & 1.118 & 13 & -6.41 & 0.000003 \\
\hline 20.17 & 1.118 & 16 & -3.73 & 0.000072 \\
\hline
\end{tabular}

Table 4: Probability table before crashing

Step 7 and 8: Table 6, 7 and 8 shows how the probability to meet the target time is increased further for different values of $p$. Using this result, project management can take their decision on crashing and probability to finish the project on target time. Extra cost $=$ Two times of $[((11,15.5,21)-(\mathrm{p}, \mathrm{p}, \mathrm{p}) \times$ $(11,15.5,21)) \times(150,250,400)]$, since in the given project network there are two critical paths after crashing. The organization cannot take those $\mathrm{p}$ factor which overshoots their budget.

\subsection{Result Discussion}

The results given in table 4 and 5 show that the probability to meet the target time is increased after crashing, but the increment is not sufficient. In table 6,7 and 8 target times are 15 days, 13 days and 16days respectively. It is observed that in each case if $\mathrm{p}$ factor (beginning from 0.99) is gradually decreased the probability value is increased till it reaches 0.999997. For a given crashed time 


\begin{tabular}{|l|l|l|l|l|}
\hline$T_{e}$ & $\sigma$ & $T_{d}$ & $Z$ & probability \\
\hline 15.67 & 0.9128 & 15 & -0.73 & 0.23 \\
\hline 15.67 & 0.9128 & 13 & -2.92 & 0.0018 \\
\hline 15.67 & 0.9128 & 16 & 0.36 & 0.64 \\
\hline
\end{tabular}

Table 5: Probability table after crashing

\begin{tabular}{|l|l|l|l|l|l|l|}
\hline$p$ & $\begin{array}{l}T_{e}(\text { New }) \\
=15.67 p\end{array}$ & $T_{d}$ & $\begin{array}{l}\sigma(\text { new }) \\
=0.9128 p\end{array}$ & $Z$ & probability & extra cost \\
\hline 0.99 & 15.51 & 15 & 0.9037 & -0.56 & 0.29 & $(33,77.5,168)$ \\
\hline 0.9 & 14.103 & 15 & 0.82152 & 1.09 & 0.86 & $(330,775,1680)$ \\
\hline 0.8 & 12.536 & 15 & 0.73 & 3.38 & 0.999928 & $(660,1550,3360)$ \\
\hline 0.75 & 11.75 & 15 & 0.68 & 4.78 & 0.999997 & $(825,1937.50,4200)$ \\
\hline 0.74 & 11.59 & 15 & 0.675 & 5.05 & 0.999997 & $(858,2015,4368)$ \\
\hline
\end{tabular}

Table 6: Affect of probability factor when target time is 15; p factor 0.75 and 0.74 are not permissible as they overshoot the budget $(1000,2000,4000)$

\begin{tabular}{|l|l|l|l|l|l|l|}
\hline$p$ & $\begin{array}{l}T_{e}(\text { New }) \\
=15.67 p\end{array}$ & $T_{d}$ & $\begin{array}{l}\sigma(\text { new }) \\
=0.9128 p\end{array}$ & $Z$ & probability & extra cost \\
\hline 0.99 & 15.51 & 13 & 0.9037 & -2.77 & 0.003 & $(33,77.5,168)$ \\
\hline 0.9 & 14.103 & 13 & 0.82152 & -1.34 & 0.0901 & $(330,775,1680)$ \\
\hline 0.8 & 12.536 & 13 & 0.73 & 0.6356 & 0.7357 & $(660,1550,3360)$ \\
\hline 0.7 & 10.97 & 13 & 0.6389 & 3.17 & 0.99931 & $(990,2325,5040)$ \\
\hline 0.64 & 10.03 & 13 & 0.5842 & 5.08 & 0.999997 & $(1188,2790,6048)$ \\
\hline
\end{tabular}

Table 7: Affect of probability factor when target time is 13 . p factor 0.7 and 0.64 is not acceptable as budget exceeded

\begin{tabular}{|l|l|l|l|l|l|l|}
\hline$p$ & $\begin{array}{l}T_{e}(\text { New }) \\
=15.67 p\end{array}$ & $T_{d}$ & $\begin{array}{l}\sigma(\text { new }) \\
=0.9128 p\end{array}$ & $Z$ & probability & extra cost \\
\hline 0.99 & 15.51 & 16 & 0.9037 & 0.542 & 0.70 & $(33,77.5,168)$ \\
\hline 0.9 & 14.103 & 16 & 0.82152 & 2.3 & 0.98 & $(330,775,1680)$ \\
\hline 0.8 & 12.536 & 16 & 0.73 & 4.74 & 0.999997 & $(660,1550,3360)$ \\
\hline 0.79 & 12.37 & 16 & 0.7211 & 5.03 & 0.999997 & $(693,1627.5,3528)$ \\
\hline
\end{tabular}

Table 8: Affect of probability factor when target time is 16

when the probability to reach the target increases the cost factor subsequently increases. Increase in the probability to meet the target is directly proportional to the cost associated with the project. The p factor depicts the cost which exceeds the budget. So the decision maker can choose the $\mathrm{p}$ factor according 
to the demand of the problem. The following graph in fig 3 depicts the results obtained.

It is observed from Graph of $\mathrm{p}$ factor and probability w.r.to a common reference, in fig 3, that slope of the probability carve is very high from the point where $\mathrm{p}$ factor is around 1 till the point where the curve of $\mathrm{p}$ factor and probability meets. Beyond this meeting point the increase rate of probability gets reduced. In other words, improvement rate of probability is very high till the meeting point, i.e., small change of $\mathrm{p}$ factor value in that region will improve the probability to finish the project on or before target time a lot. And beyond that point, probability gets saturated. So we can name the first region where slope is higher in the probability curve as active region and the other part as passive region. Choosing $\mathrm{p}$ factor in the active zone will always be cost-efficient as small reduction in $\mathrm{p}$ factor, thereby the extra cost, will noticeably improve the probability. Another characteristics is observed from Graph of $\mathrm{p}$ factor and probability w.r.to a common reference. Considering the target times as a little lower and higher than the normal crashed time respectively, the meeting point shifts a little down and up with respect to that obtained for the crashed time as target time. As a result, this can be concluded that the improve rate of probability is higher for the target times lower than the crashed time compared to the higher ones.

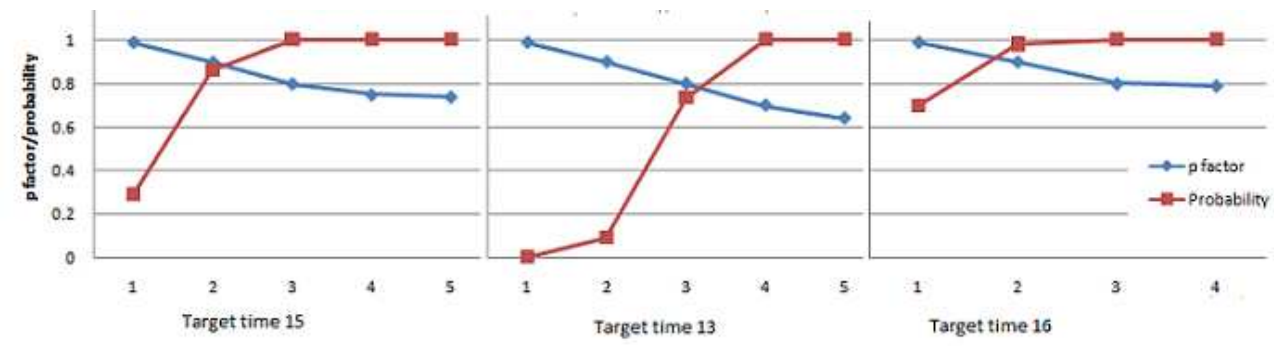

Figure 3: p factor and probability w.r.to a common reference

\section{Conclusion}

In this paper, how a project can be crashed using fuzzy activity time and fuzzy cost to express uncertainty and vagueness has been shown and $\mathrm{p}$ factor has been introduced and applied to increase the probability to meet the target time after crashing. 


\section{References}

[1] S.Suresh Babu, Y.L.P. Thorani and N.Ravi Shankar, Ranking Generalized Fuzzy Numbers using centroid of centroids, International Journal of Fuzzy Logic Systems, 2 (2012), 17-32, doi: 10.5121/ijfls.2012.2302.

[2] N Ravi Shankar, V Sireesha and P Phani Bushan Rao, An Analytical Method for Finding Critical Path in a Fuzzy Project Network, Int. J. Contemp. Math. Sciences, 20 (2010), 953-962.

[3] Shih-Pin Chen and Yi-Ju Hsueh, A simple approach to fuzzy critical path analysis in project networks, Applied Mathematical Modelling, 32 (2008), 1289-1297, doi: 10.1016/j.apm.2007.04.009.

[4] Stefan Chanas and Pawel Zielinski, Critical path analysis in the network with fuzzy activity times, Fuzzy Sets and Systems, 122 (2001), 195-204, doi: 10.1016/S01650114(00)00076-2.

[5] Ahmed Farouk Abdul Moneim,Expected Delays in Completing Projects under Uncertainty, Jordan Journal of Mechanical and Industrial Engineering, 2 (2008), 65-69.

[6] Stephan Foldes and Franois Soumis, PERT and crashing revisited: Mathematical generalizations, European Journal of Operational Research, 64 (1993), 286-294, doi: 10.1016/0377-2217(93)90183-N.

[7] Ten Wei Peng, Mustafa bin Mamat and Yosza bin Dasril, An Improvement of Numerical Result of Crashing CPM/PERT Network, Journal of Science and Technology, 2 (2010), $17-32$.

[8] I.M. Premachandra, A Goalprogramming Model for Activity Crashing in Project Networks, Journal of Science and Technology, 13 (1993), 79-85.

[9] Y. A. Ghaleb and M. M Adnan, Crashing PERT network using mathematical programming, International Journal of Project Management, 19 (2001), 181-188, doi: 10.1016/S0263-7863(99)00061-7.

[10] Gary Mitchell and Ted Klastorin, An effective methodology for the stochastic project compression problem, IIE Transactions, 39, (2007), 957-969, doi: 10.1080/07408170701315347.

[11] Mustafa bin Mamat, Ten Wei Peng, Yosza bin dasril and Ismail bin Mohd, MultiDimensional Nonlinear Knapsack Problem for crashing CPM/PERT, World Applied Sciences Journal, 13 (2011), 341-346.

[12] A. Nagoor Gani, A New Operation on Triangular Fuzzy Number for Solving Fuzzy Linear Programming Problem, Applied Mathematical Sciences, 11, (2012), 525-532.

[13] Priti Singh, Florentin Smarandache, Dipti Chauhan and Amit Bhaghel, A unit based crashing pert network for optimization of software project cost, Int. J. Contemp. Math. Sciences, doi: 10.1.1.310.187.

[14] Ajiboye Sule Adegoke, Measuring Process Effectiveness Using Cpm/Pert, International Journal of Business and Management, 6 (2011), doi: 10.5539/ijbm.v6n6p286.

[15] Komesh Sahu and Meenu Sahu, Cost \& time and also minimum project duration using alternative method, International Review of Applied Engineering Research, 4 (2014), 403-412. 
[16] P.K. Suri and Bharat Bhushan, Simulator for Optimization of Software Project Cost and Schedule, cost, risk, and quality criteria, Applied Mathematical Sciences, 4 (2008), 1030-1035. 